$\mathbb{T}$ periodica polytechnica

Transportation Engineering

$36 / 1-2$ (2008) 15,19

doi: $10.3311 /$ pp.tr.2008-1-2.03

web: http://www.pp.bme.hu/tr

(c) Periodica Polytechnica 2008

RESEARCH ARTICLE

\section{Development and evaluation of a} Fuzzy-based Microscopic Vehicle-following model

\author{
Tamás Bécsi / Tamás Péter
}

Received 2007-03-03

\begin{abstract}
The article presents a Fuzzy-Sugeno type microscopic follower model from the building of the model through a brief description to the evaluation of it. Four other models mentioned in the literature are used for the investigation of the model giving a basis of comparison for the evaluation of the model in question. The model implements a partition of two of the three typical parameters (follower distance, speed, difference in speed) to achieve the required output.
\end{abstract}

\section{Keywords}

Microscopic traffic modelling $\cdot$ Sugeno-Fuzzy systems

\section{Tamás Bécsi}

Department of Control and Transport Automation, BME, Bertalan L. u. 2. H1111 Budapest, Hungary

e-mail: becsi.tamas@mail.bme.hu

\section{Tamás Péter}

Department of Control and Transport Automation, BME, Bertalan L. u. 2. H1111 Budapest, Hungary

e-mail: peter@kozlek.bme.hu

\section{Introduction}

In the research process the determination of the parameters of five microscopic traffic models take place. The principle of choosing these five investigated models is, that the models should describe the traffic model in the same depth, what means that the "microscopic" level of each model is the same, and the numbers of the input parameters are nearly equal. So the models are in each case microscopic models with a classical system of conditions, where the "vehicle-driver" is one system, the input parameters are always the motional characteristics of the vehicles following and being followed, and the output is the acceleration applied in a given time of reaction. (The original output of the Gipps-model is speed, but as the restrictions of the models are a maximal acceleration/deceleration, this model implies the acceleration parameter as well.) These models are:

- Gazis-Herman-Rothery (GHR); [2]

- Gipps (Aimsun); [4]

- Wiedemann (Vissim); [3]

- Simple Fuzzy model; [1]

- Model based on Fuzzy states [present document]

The investigation of the Wiedemann model shows that covering the fundamental sets of the microscopic model by several mutually independent sets approximate the attributes of real-life driving relations very well. One of the draw-backs of the Wiedemann model is, nevertheless, that the resulting output function is not continuous at the limits of state given. In cases of "undisturbed" vehicle following the experimental results are continuous functions, though.

It is reasonable to look for a behavioural model that offers continuous gradation instead of a gradation in leaps between state limits. The model to be set up should therefore include an appropriate number of follower models in accordance with the number of the different states, and it should make sure that the gradation between the states is smooth and continuous. An appropriate tool can be the covering of the fundamental set by Fuzzy-sets and applying a basis of rules. While creating a model one has to take certain factors into consideration: 
1 The model, as it gives the backbone of the simulation, cannot be too complicated, therefore the fundamental set should be covered by the smallest possible number of fuzzy sets.

2 As a consequence one should - if it is possible - "fuzzificate" as few input parameters as possible as the final resulting number of sets is equal to the product of the number of the sets in the different dimensions.

3 One has to find an appropriate relation on the multidimensional fundamental set that provides the appropriate creation of the multi-dimensional fuzzy set.

4 Such a simple function is to be found for the rule of deduction of the different states that is fast enough in the case of several calculations as well.

5 The sets should cover the whole sample set so that for each input there should be a rule generating an output for it.

For this task, the Sugeno basic type of the Fuzzy deductions can be an appropriate tool. Considering the classical approach, the three-dimensional approach of dividing the sample set into follower-distance, speed and difference in speed seems to be obvious.

The GHR model indicates that it is useful to divide the difference in speed into two distinct parts: approaching and leaving.

Considering the second point it is necessary to leave out one or two dimensions for the fuzzification of the detection sample set. Based on one of the basic attributes of the Wiedemann model we can distribute the follower distance into two parts: closer and farther regions. Naturally the estimation of the extent and dangerousness of the follower distance is greatly dependent on the speed of the vehicle and the speed of the vehicle in front of it, we can use this attribute to make the parameters of the fuzzy sets to the follower distance dependent on the speed of the vehicle.

\subsection{Takagi-Sugeno-Kang type state model}

Considering the practical planning suggestions, the experiences of the simple Fuzzy-model and using the basic concepts of "Action Point" models a new model can be created that is distributing the vehicle states in advance.

During the investigation of models and the creation of new ones it became obvious that detecting states are to be put up in accordance with the follower distance and difference in speed, with the restriction that the evaluation of the follower distance should be a function on speed. Therefore the following four states can be built:

\subsubsection{Building up the model}

Fuzzification of the speed is omitted in this model. Instead the determination of the follower distance expected can take place in a similar way like in the case of the first model using an additional predictional term dependent on the difference of speed
Tab. 1. The distribution of the state space of following

\begin{tabular}{|c|c|c|c|}
\hline & & \multicolumn{2}{|c|}{ Difference in speed } \\
\hline & & Leaving & Approaching \\
\hline $\begin{array}{l}Ð \\
\frac{0}{\pi} \\
\frac{\pi}{0} \\
\frac{0}{0}\end{array}$ & 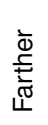 & $\begin{array}{l}\text { The effect of the fol- } \\
\text { lowed vehicle on the } \\
\text { behaviour is weak }\end{array}$ & The followed vehicle is regarded \\
\hline $\begin{array}{l}\text { ఏँ } \\
\sum_{0}^{\circ} \\
\overline{0}\end{array}$ & $\begin{array}{l}\infty \\
\infty \\
\text { 은 }\end{array}$ & $\begin{array}{l}\text { The followed vehicle } \\
\text { is regarded }\end{array}$ & $\begin{array}{l}\text { The followed vehicle is regarded } \\
\text { intensely (nearly exclusively). } \\
\text { (Braking, probable situation of } \\
\text { taking over and changing lanes) }\end{array}$ \\
\hline
\end{tabular}

and the reaction time. The function of the new term is, instead of generating a follower distance value for the present state, to take the followed vehicle and own speed into consideration:

$$
D_{\text {foll }}^{*}=v\left(p_{1} r_{1}+p_{2}\right)+p_{3} r_{1}+p_{4}+\Delta v \Delta T,
$$

Where $D_{\text {foll }}^{*}$ is the follower distance expected $\mathrm{p}\left[\mathrm{p}_{1}, \mathrm{p}_{2}, \mathrm{p}_{3}, \mathrm{p}_{4}\right]$ model-parameter, $r_{1}$ the vehicle's own attribute, $\Delta v \Delta \mathrm{T}$ distance factor originated from the difference in speed and reaction time.

Follower distance sets can be defined in simple trapezoid shaped sets.

$$
\begin{aligned}
& \text { Close }: D F_{1}= \\
& \left\{\begin{array}{lll}
1, & \text { if } \quad d x-D_{\text {foll }}^{*}<a_{1} \\
\frac{\left(b_{1}-d x\right)}{\left(b_{1}-a_{1}\right)}, & \text { if } & a_{1} \leq d x-D_{\text {foll }}^{*} \leq b_{1} \\
0, & \text { if } & d x-D_{\text {foll }}^{*}>b_{1}
\end{array}\right\} \\
& \text { Farther }: D F_{2}= \\
& \left\{\begin{array}{lll}
1, & \text { if } & d x-D_{\text {foll }}^{*}<a_{2} \\
\frac{\left(d x-a_{2}\right)}{\left(b_{2}-a_{2}\right)}, & \text { if } & a_{2} \leq d x-D_{\text {foll }}^{*} \leq b_{2} \\
0, & \text { if } & d x-D_{\text {foll }}^{*}>b_{2}
\end{array}\right\}
\end{aligned}
$$

$$
\begin{aligned}
& \text { Approaching: } D V_{1}= \\
& \left\{\begin{array}{lll}
1 & \text { if } \quad d v<c_{1} \\
\frac{\left(d_{1}-d v\right)}{\left(d_{1}-c_{1}\right)}, & \text { if } \quad c_{1} \leq d v \leq d_{1} \\
0 & \text { if } \quad d v>d_{1}
\end{array}\right\} \\
& \text { Leaving: } D V_{2}= \\
& \left\{\begin{array}{lll}
1 & \text { if } d v<c_{2} \\
\frac{\left(d v-c_{2}\right)}{\left(d_{2}-c_{2}\right)}, & \text { if } \quad d_{2} \leq d v \leq d_{2} \\
0, & \text { if } d v>d_{2}
\end{array}\right\}
\end{aligned}
$$

The condition of the covering of the whole sample set is that the single sets should overlap, in this case therefore the inequalities

$$
a_{1}<b_{2} \quad \text { and } \quad c_{1}<d_{2}
$$

should be true. If the conditions

$$
a_{1}=a_{2} ; b_{1}=b_{2} ; c_{1}=c_{2} ; d_{1}=d_{2}
$$

are true then

$$
\begin{array}{ll}
D V_{1}(d v)+D V_{2}(d v)=1 & \forall d v \\
D F_{1}(d x)+D F_{2}(d x)=1 & \forall d x
\end{array}
$$


that is the sets of the different dimensions form a Ruspinpartition. Four conditions can be created by four sets that are the intersections of two sets of different dimensions:

$$
\begin{aligned}
& \mathrm{A}=\left\{A_{i} ; i \in[1,4]\right\} \\
& A_{1}(d x, d v)=t\left(D F_{1}(d x), D V_{1}(d v)\right) \\
& A_{2}(d x, d v)=t\left(D F_{1}(d x), D V_{2}(d v)\right) \\
& A_{3}(d x, d v)=t\left(D F_{2}(d x), D V_{1}(d v)\right) \\
& A_{4}(d x, d v)=t\left(D F_{2}(d x), D V_{2}(d v)\right)
\end{aligned}
$$

where $\mathrm{A}_{k}$ is the two-dimensional fuzzy set of state, $\mathrm{t}\left(\mathrm{DF}_{i}, \mathrm{DV}_{j}\right)$ is the t-norm forming the intersection. " $\mathrm{A}$ " is the fuzzy partition of the whole sample set. If we define intersection as a Zadehtype of intersection that is

$$
t\left(D F_{1}(d x), D V_{1}(d v)\right)=\min \left(D F_{1}(d x), D V_{1}(d v)\right),
$$

the sets of states fulfil the following figure, but it is important to mark that this two-dimensional partition can no longer be treated as a Ruspin partition:

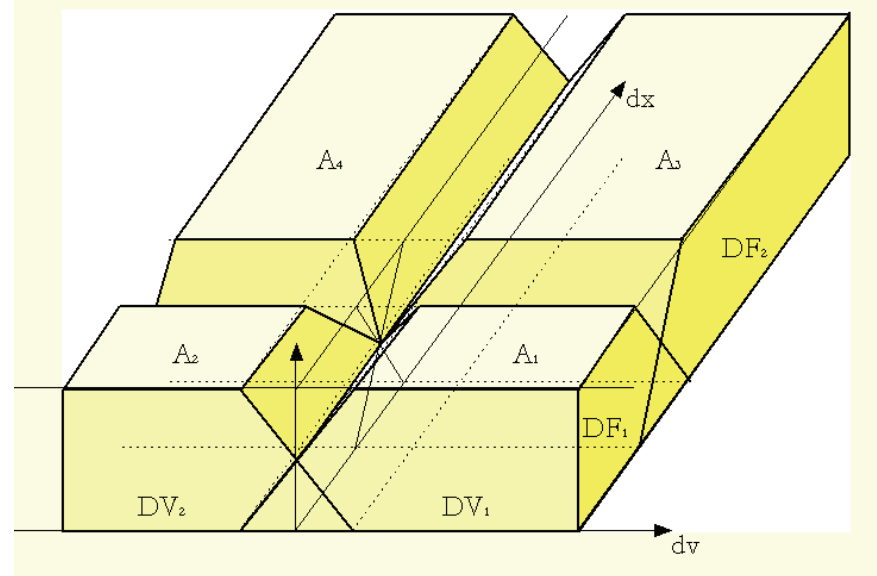

Fig. 1. State sets formed by the Zadeh-type $t$-norm

\subsubsection{Defuzzification}

Based on the position occupied in the sample set, the system should generate an output that is the driver has to accelerate according to the circumstances. Continuity of the acceleration values can be made sure by any deduction of the Sugeno type. If the values of the terms of the partition are

$$
w_{i}=A_{i}(d x, d v) ; \forall i
$$

then, the general (Takagi-Sugeno-Kang) form of deduction is:

$$
y=\frac{\sum_{1=1}^{n} w_{i} * f_{i}(d x, d v, v)}{\sum_{1=1}^{n} w_{i}},
$$

where $\mathrm{f}_{i}(\mathrm{dx}, \mathrm{dv}, \mathrm{v})$ is the output function belonging to state $i$.

In the creation of the deduction function two basic approaches are possible:
Implementation of an existing model or models state by state. This approach can be an excellent solution as model parameters can be determined not for the whole state space but for several - in this case four - separate states, and so the model is more precise with a minimal extra in calculations.

The decision process of the states can be solved using a new "soft computing" algorithm as well. In this case we get a twolevel fuzzy deduction. The model built also uses this possibility so in the followings we will go into details.

Four states are given, therefore, which are to be created using the appropriate deduction function $f_{i}(d x, d v, v)$. The state " $i$ " is to be partitioned according the aforementioned model:

$$
P S^{i}=P S_{j}^{i}(\Delta v) ; \quad P F^{i}=P F_{k}^{i}\left(\Delta x-D_{f o l l}^{*}\right)
$$

$$
j \in(1 . . n), k \in(1 . . m),
$$

where $P S^{i}$ and $P F^{i}$ are the partition of the difference in speed and follower distance in state " $\mathrm{i}$ ", $\Delta \mathrm{v}$ and $\Delta \mathrm{x}$ are difference in speed and actual follower distance, $\mathrm{n}$ and $\mathrm{m}$ are the measures of the partition.

$$
\begin{aligned}
& a_{d s p e}^{i}=\frac{\sum_{j=1}^{n} P S_{j}^{i}(\Delta v) A_{i}^{i, v}}{\sum_{j=1}^{n} D S_{j}^{i}(\Delta v)} ; \quad a_{d f o l l}=\frac{\sum_{k=1}^{m} D F_{k}^{i}\left(\Delta x-D_{\text {foll }}^{*}\right) A_{j}^{i, f}}{\sum_{k=1}^{m} D F_{k}^{i}\left(\Delta x-D_{\text {foll }}^{*}\right)} \\
& j \in(1 . . n) \\
& k \in(1 . . m) \\
& a_{\text {med }}^{i, 1}=a_{d s p e}^{i} r_{2}+a_{d f \text { oll }}^{i}\left(1-r_{2}\right) \quad ; \quad r_{2} \in[0 ; 1] \\
& a_{\text {med }}^{i, 2}=\left(p_{5}+p_{6} r_{2}\right) a_{\text {med }}^{i, 1} \\
& f_{i}=\left\{\begin{array}{l}
a_{\max } a_{\text {med }}^{i, 2} ; a_{\text {med }}^{i, 2}>0 \\
a_{\min } a_{\text {med }}^{i, 2} ; a_{\text {med }}^{i, 2} \leq 0
\end{array}\right\}
\end{aligned}
$$

Table 1 shows an outcome of an acceleration belonging to a possible speed and driver parameters:

\subsubsection{Identification, model evaluation}

The identification of the models took place using a data set acquired by the refinement of microscopic databases loaded from the page of "Next Generation Simulation Community" [5]. The search for the optimum in its final form was done by a unigenous genetic algorithm assisted by a simulated annealing that is:

- Elitist (in 5\%);

- Has a real gene-representation;

- Modifies the fitness value with the function $\operatorname{random}(\mathrm{T})^{*}$ of the simulated annealing;

- Thus realizing the selection with absolute sequencing;

- Applying the mutation in a radius of "random(T)"*;

- Applying a complete recombination;

- Has a constant size of population.

* - uniform distribution in the interval [-T; T] 
Fig. 2. Acceleration diagram belonging to $5 \mathrm{~m} / \mathrm{s}$ of the state divided fuzzy model

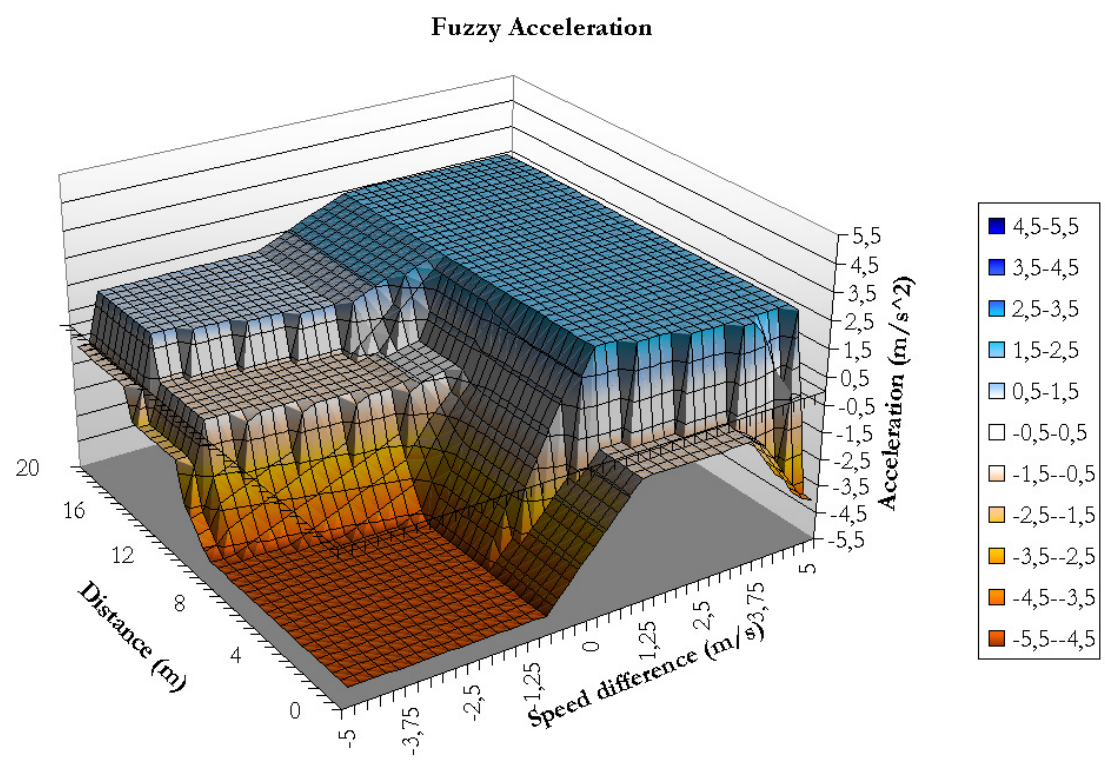

Fig. 3. Evaluation of the microscopic and macroscopic attributes of the Fuzzy model

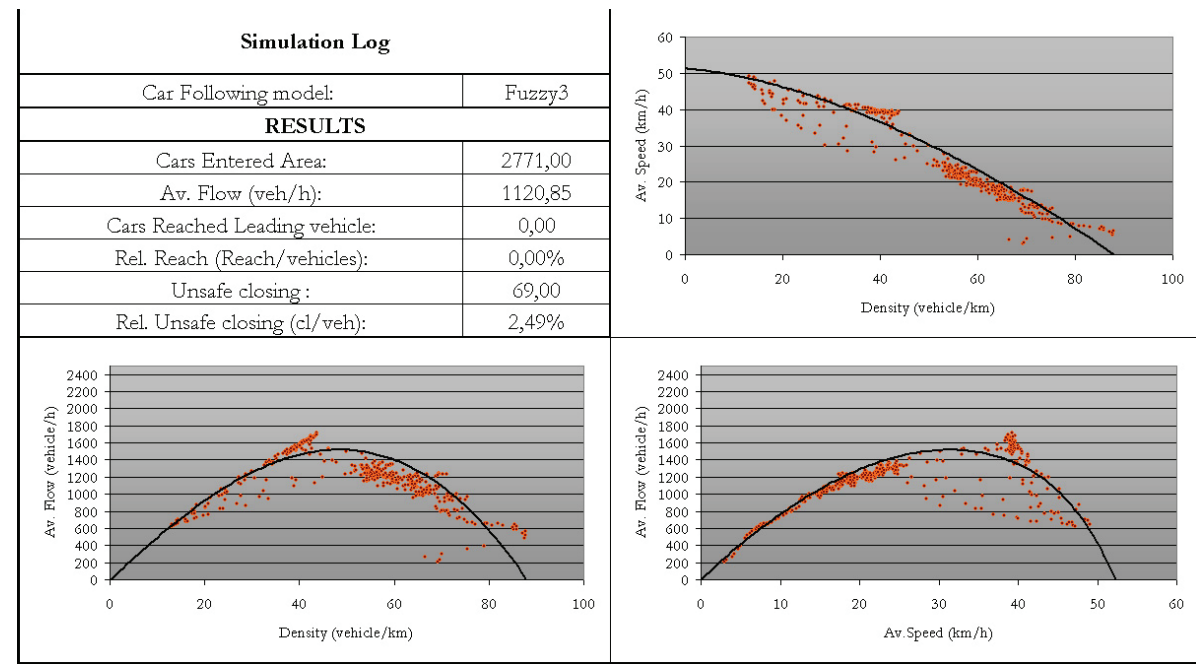

Fig. 4. General evaluation of the model achievements

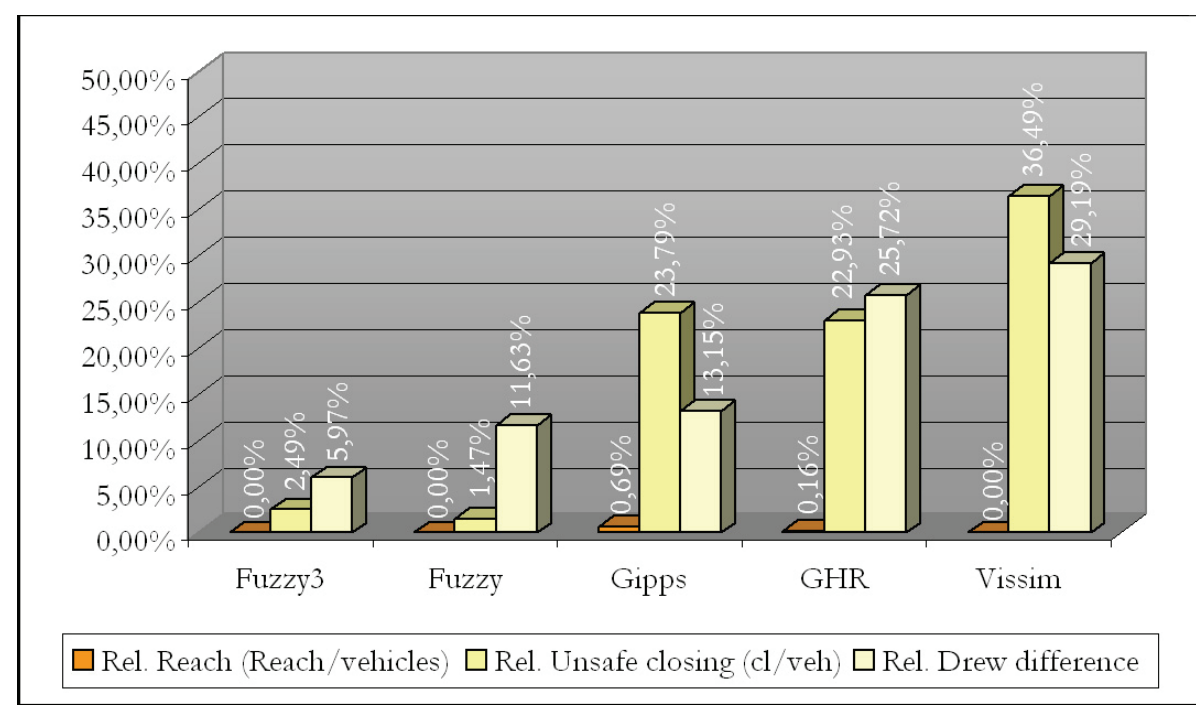

The result of the fuzzy model is represented in Fig. 3. The model can be regarded as totally stabile in the microscopic level. It is true because, apart from the Wiedemann model, this is the only model possessing a reaction-free platform belonging to a stationary flow, and so the models can apply $0 \mathrm{~m} / \mathrm{s}^{2}$ acceleration not only in a given input point but on a given region, and so the behaviour of the driver is more relaxed. The model approximates the macroscopic curve well.

\subsubsection{Comparing models}

Fig. 4 shows the diagrams of the general evaluation of the model achievements. For the comparison of the models three 
basic parameters are used. Among these next to the two essential parameters - the ratio of reach and the macroscopic difference from the Drew model - the ratio of unsafe closing is represented as well.

From the microscopic point of view, one can accept the two Fuzzy models and the Wiedemann model as neither will represent a critical overshoot, the flow of traffic can be considered as calm. In this approach the Gipps model is the odd one out because of its great number of overshoots.

In microscopic behaviour again, the two Fuzzy models and the Gipps model have an acceptable achievement, what means they approximate the actual curve of traffic. Wiedemann (Vissim) model is the odd one out because of its excess goal for safety.

\section{Modelling, identification and the conclusion of the simulation results}

It is important to note that the present results have an exemplary importance regarding the identification of models, that is that in the case of the determination of model parameters one has to be careful in selecting the data sequences used for the identification.

These data sequences should include more critical situations so that when implemented in the model simulation it should behave more stabile. Measuring such data sequences is very tiresome as it is difficult to capture such situations because of their rarity.

Another solution could be to generate such a critical situation in certain circumstances, but in this case only controlled measuring could be considered because of traffic safety reasons, nevertheless in this case the driver of the vehicle measured would be aware of being measured, and the critical situation and thus it would effect his behaviour and endanger the reach of the goal of the measuring.

A further possibility would be to run a control-simulation parallel to the determination of model parameters, that would evaluate models not just regarding measured values but parallel to it - viewed globally - right during its operation. This possibility can be regarded only in theory as an investigation presented in this chapter that would examine microscopic and macroscopic features as well would take $70 \mathrm{~s}$ (considering a $2 \mathrm{GHZ}, 32$ bit processor). This access of time would be out of question in each step of the search for optimum even if applying the elitist approach, where the examination regards the individual with the best fitness values.

It is important to examine the circumstances of the determination of model parameters. Both literature and this document insist on an approximation of measured microscopic characteristics, and require a model resulting that adjusts to stabile traffic situations and macroscopic attributes measured or assumed to be ideal. Nevertheless there would be a model determining structure created that would use a totally different approach. In this case the stability criteria required and the macroscopic be- haviour would give the goodness value of the model, that would be evaluated on an examined section or even an examined net with a simulation in a way, that the whole vehicle population would be regarded as a subject of the examination. As vehicles after a time are sure to leave the net the change in generations would be automatic. The survival chance of the genes of a vehicle would be affected by its own defects and the goodness of those macroscopic attributes in which the effects of the vehicle appear. It is clear that in this structure the fitness of a vehicle is indirectly affected by the behaviour of the other vehicles but it can lead to a result in evolutional algorithms conveyed in several different areas with analogous conceptions. The method can result in a very processor challenging application and it is partly the reason why such an application is not mentioned in the literature.

\section{References}

1 Bécsi T, Péter T, Forgalmi modellek identifikációja a videó-mérésból kinyert paraméterhalmaz alapján, (2006 1.1-K1-4)”, EJJT RET Kutatási jelentés, 2006.

2 Gazis DC, Herman R, Potts RB, Car following theory of steady state traffic flow, Operation Research 7 (1959), 499-505.

3 Wiedemann R, Reiter U, Microscopic Traffic Simulation: The Simulation System MISSION. Background and Actual State, CEC Project ICARUS (V1052) Final Report, 1992. Appendix A. CEC.

4 Gipps PG, A Behavioural Car-following Model for Computer Simulation, Transportation Research Part B, Issue 2, 1981, pp. 105-111.

5 Next Generation Simulation Community, available at http://www.ngsim. fhwa.dot.gov/ 